\title{
Computational Techniques for Infectious Diseases and
}

\section{Clinical Research}

\author{
Mannar Jawahar Ponnuswamy ${ }^{1}$, Kamalanand Krishnamurthy ${ }^{2}$ \\ ${ }^{1}$ Centre for University Industry Collaboration, Anna University, Chennai 600025, India \\ 2 Department of Instrumentation Engineering, MIT Campus, Anna University, Chennai 600044, India
}

The unpredictable nature of infectious diseases has been a source of fear and superstition since early times. The purpose of introducing mathematics in the field of infectious diseases is to understand the dynamics of infections and for analysis [1]. The first mathematical model of an infectious disease was proposed by Daniel Bernoulli, a Swiss Mathematician and Physicist. In 1760, Daniel Bernoulli developed a differential equation model to quantitatively analyze how cowpox inoculation affects the spread of smallpox. Further, Bernoulli used the model to assess the practical advantages of a vaccination program [2, 3].

Over the years, the use of mathematical and computational models for understanding the dynamics of the infection, for analyzing the spread of diseases and for establishing the effectiveness of vaccines, has greatly revolutionized the study of infectious diseases and epidemic outbreaks [4]. The computational techniques for analyzing infectious diseases are diverse and growing rapidly. Some techniques have been newly developed, whereas others have been derived from diverse fields including dynamical systems, stochastic processes, statistical physics, graph theory, statistics, operations research and high-performance computing [5].

Recently, several computational methods such as image processing, numerical methods, optimization, statistics, artificial intelligence etc. have been successfully employed for biomedical analysis and medical diagnostics. Such tools have proved to be valuable for treatment and surgery planning, design of biosensors and development of decision support systems.

The European Journal for Biomedical Informatics (EJBI), being an official journal of the European Federation for Medical Informatics, offers an excellent forum for sharing and understanding of interdisciplinary scientific knowledge between the biomedical researchers and the healthcare industry. Being a multilingual journal, EJBI also ensures the wide reach of information on an international scale.

This special issue of EJBI entitled "Applications of Computational Techniques in Infectious Diseases and Clinical Research" gathers the authors' effort to bring relevant scientific and technological discussion about the applicability of computational methods such as image processing, intelligent computing, finite element methods, etc. in multidisciplinary areas of infectious diseases and clinical research.

This issue comprises eight excellent contributions which depict ideas applied in diverse areas such as intelligent biosensor development, infectious diseases such as HIV and tuber- culosis, analysis of clinical signals and images, and clinical biomechanics.

Tuberculosis is a highly infectious disease and is a major cause of death in underdeveloped and developing countries. In the paper presented by Priya and Srinivasan, an automated decision support system based on evolutionary extreme learning machines and sputum smear images has been developed for diagnosis of Tuberculosis infection.

In recent years, the spread of HIV/AIDS has become a global problem and is considered to be the most severe health crisis of modern times. In the article submitted by Senbagamalar and Baskar Babujee, the authors have analyzed the anti HIV activity of Quinolone Carboxylic acids using graph theory.

Next, in the research article presented by Subashini et al., the authors have extensively analyzed the antimicrobial effects of the selected herbal plants such as Solanum xanthocarpum, Solanum nigrum and Helianthus annuus, using digital image processing techniques.

In the next paper authored by Ganapathi et al., the authors have used finite element methods for design and analysis of a hip implant model for surgical planning. The authors have further optimized the implant for improving patient safety.

Breast cancer is one of the most common malignancy in women and is a continuing threat worldwide. In the paper presented by Yuvaraj and Ragupathy, a computer aided method for segmentation and classification of mass in mammographic images has been developed using a hybrid neurofuzzy system.

In the next article presented by Najumnissa and Rangaswamy, a detailed comparison on the classification performance of intelligent computing techniques such as back propagation networks, adaptive neuro-fuzzy inference system, particle swarm optimization based neural networks and radial basis function neural networks, for classification of normal and abnormal EEG signals, has been performed.

The paper authored by Innocent et al., describes a computer aided semen quantification technique for analyzing the quality of sperm in men. At present, infertility has become a major concern in marital relationships and an automated system for evaluation of sperm quality is highly useful for fast and efficient analysis of infertility in men.

In the final paper of this special issue, presented by Durai Arun et al., the authors have developed an image based microtiter plate reader system for 96-well format fluorescence 
assays, using a blue light excitation and a k-means classifier. Very promising results have been obtained and this interesting work appears to be of high clinical importance.

This joint Indo-European effort clearly magnifies the applicability and importance of computational methods in the fields of infectious diseases and clinical research. We are indebted to the Editor-in-Chief, Prof. Dr. Jana Zvárová and the Executive Editor, Dr. Petra Prečková for their valuable support. The Editors would like to thank the Reviewers of this special issue, for their excellent reviews and comments.

\section{References}

[1] Choisy M, Guégan J.-F, Rohani P. Mathematical Modeling of Infectious Diseases Dynamics. Chapter 22. Encyclopedia of In- fectious Diseases: Modern Methodologies, by M.Tibayrenc. John Wiley Sons. 2007.

[2] Bernoulli D. Essai d'une nouvelle analyse de la mortalité causée par la petite vérole et des avantages de l'inoculum pour la prévenir. Mém Math Phys Acad Roy Sci Paris 1760; 1-45.

[3] Dietz K, Heesterbeek JAP. Daniel Bernoulli's epidemiological model revisited. Mathematical Biosciences 2002; 180: 1-21.

[4] Hollingsworth TD. Controlling infectious disease outbreaks: Lessons from mathematical modelling. Journal of Public Health Policy 2009; 30(3): 328-341.

[5] Dimitrov NB, Meyers LA. Mathematical Approaches to Infectious Disease Prediction and Control. Tutorials in Operations Research, INFORMS 2010; 1-25. 